\title{
Die Rolle von Klein- und Mittelunternehmen im politischen Kampf um TTIP. Eine hegemonietheoretische Betrachtung
}

\begin{abstract}
Simon Theurl*
Zusammenfassung

Klein- und Mittelunternehmen (KMU) werden seit dem Frühjahr 2015 von der Europäischen Kommission als die Gewinner von TTIP und CETA dargestellt. Diese Aussage ist jedoch stark umstritten, nicht zuletzt, weil die ökonomischen Effekte von Handelsliberalisierung ambivalent sind: Tendenzen zur Monopolisierung sind ebenso denkbar (modellierbar) wie der Anstieg an Produkt-Vielfalt und Effizienzgewinnen. Befürworter*innen von TTIP stellen jedoch ausschließlich mögliche Vorteile für KMU in den Vordergrund. Das wird in diesem Paper als ein strategisches Manöver interpretiert, welches darauf abzielt, Zustimmung für die Umsetzung dieser Handelsabkommen, die weit über die Reduktion von Zöllen hinausreichen, zu erhalten. Aus hegemonietheoretischer Perspektive sollen Herrschaftsverhältnisse in den Vordergrund gestellt werden und soll argumentiert werden, dass TTIP und CETA Projekte im Interesse eines „transnationalen Blocks" sind.
\end{abstract}

Schlagwörter: TTIP, CETA, Hegemonie, KMU, Klein- und Mittelunternehmen

SMEs in the political struggle against TTIP.

An analysis from a hegemony-theoretical perspective.

\begin{abstract}
Since spring 2015 the European Commission began to argue that small and medium enterprises (SMEs) would be the real winners from TTIP and CETA. This statement is strongly combated as the economic effects of trade liberalization are ambiguous: Theoretically trade liberalization can lead to both, an increase of monopolistic competition, or an increase of variety and efficiency because of increased competition. But supporter of TTIP only put forward the possible benefits for SMEs. In this paper this is interpreted as a strategic maneuver to gain support for the implementation of the so called "deep trade agreements" TTIP and CETA. From a hegemony theoretical perspective power-relations are analyzed in this paper and it will be argued that TTIP and CETA are actually projects which reflect the interest of a "transnational block".
\end{abstract}

Keywords: TTIP, CETA, hegemony, SME, small and medium enterprises

${ }^{*}$ Theurl Simon, Freischaffender Wissenschaftler und Lektor, ATTAC, BEIGEWUM. E-Mail: simon_theurl@hotmail.com 
Seit dem offiziellen Start der Verhandlungen über das Freihandelsabkommen Transatlantic Trade and Investment Partnership (TTIP) im Sommer 2013 spitzt sich die aktuelle handelspolitische Debatte innerhalb der europäischen Zivilgesellschaft um die Themen TTIP, Comprehensive Economic and Trade Agreement (CETA) und Trade in Services Agreement (TiSA) zu. Wie schon bei vergangenen Handelsabkommen sind auch diesmal die geplanten Gesetzesänderungen Ursache für globale Proteste ${ }^{1}$ (Eberhardt 2014b). Doch trotz des außergewöhnlich starken Engagements in der Zivilgesellschaft (getragen von dem Engagement von NGOs, Gewerkschaften und Kammern), versuchen Befürworter*innen des Abkommens in den verschiedensten politischen Positionen ihr Programm möglichst kompromisslos voranzutreiben (ebd.). In dieser Auseinandersetzung um die Umsetzung der neuen Handelsagenda markiert(e) die - seit ca. April 2015 verstärkt geäußerte - Aussage, TTIP käme besonders Klein- und Mittelunternehmen (KMU) zugute, eine inhaltliche Erweiterung der Pro-TTIP-Argumentation, welche stark von der Europäischen Kommission (EK) propagiert wird und sich in der Argumentation anderer Pro-TTIP-Fraktionen wiederfinden ${ }^{2}$ lässt. Dabei wurde die Aussage, TTIP käme vor allem KMU zugute, selbst $\mathrm{zu}$ einem umstrittenen Themenpunkt in der Debatte über TTIP und führte schließlich dazu, dass KMU selbst als politische Gruppe aktiviert und aktiv wurden.

Die Auseinandersetzung über TTIP wurde somit nicht nur inhaltlich erweitert, sondern erzeugte zugleich auch eine weitere Bruchlinie zwischen den Kapitalfraktionen, nämlich zwischen transnationalen Großkonzernen (TNK) und KMU. Eine Bruchlinie, die selbst umkämpft ist: nämlich entlang der Frage, ob KMU besonders von TTIP profitieren können. Dabei sind die Motive der Klein- und Mittelunternehmer*innen, gegenüber TTIP in Opposition zu gehen, sehr vielseitig

1 Die Liste der Organisationen des Stopp TTIPBündnisses geben einen Überblick über die Streuung der zivilgesellschaftlichen Organisationen, die sich aktiv gegen TTIP und CETA in Europa einsetzen. Online: https://stopttip.org/de/unterstutzerorganisationen/ [31. Oktober 2016]; Das Netzwerk „Seattle to Brussle“ vernetzt die Proteste zwischen Europa und den USA. Online: http://www.s2bnetwork. org/cat/activities/ [31. Oktober 2016]; Die Suchbegriffe „Protest TTIP“ stoßen auf 535.00o Ergebnisse in 0,5 Sekunden [31. Oktober 2016]

2 In Bezug auf die Auswirkungen von TTIP auf KMU decken sich beispielsweise die Argumente der Wirtschaftskammer Österreich (WKO) mit jenen der EK. und reichen weit über die möglichen ökonomischen Konsequenzen und damit über die Implikationen auf die handelnden Akteur*innen in ihren Rollen als Unternehmer*innen hinaus (Theurl/Grumiller 2016).

Um TTIP unter dem Aspekt von Herrschaftsverhältnissen $\mathrm{zu}$ analysieren und $\mathrm{zu}$ interpretieren, wird die politische Auseinandersetzung über TTIP im Folgenden als Ringen um Hegemonie betrachtet. Um Herrschaft hegemonial aber möglichst ohne Zwang auszuüben, bedarf es eines möglichst breiten Konsenses. Dieser wird somit selbst zu einem wesentlichen Aspekt politischer Auseinandersetzungen. Um also für TTIP (und andere politische Initiativen) eine möglichst breite Zustimmung zu erlangen, ist es notwendig, dass, sobald die Thematik öffentlich behandelt wird, sich möglichst viele Interessensgruppen in dem geplanten Projekt vertreten sehen.

In diesem Licht können die bereits zu Beginn der Debatte über TTIP und CETA in Aussicht gestellten positiven makroökonomischen Wachstumseffekte als eine konsensstiftende Strategie verstanden werden. Konkret wurden dabei positive Effekte von TTIP mit eigens angefertigten Studien untermauert, die von der EK bei den Think-Tanks Center of Economic Policy Research ( Francois et al. 2013) sowie bei ECORYS in Auftrag gegeben wurden. Doch schon zu Beginn der politischen Auseinandersetzungen um TTIP wurde von Akteur*innen der Zivilgesellschaft (AK, NGOs wie ATTAC, Gewerkschaften usw.) gezeigt, dass die Versprechen von neuen Jobs, Wachstum, usw. ... auf Sand gebaut sind. In der von der Zivilgesellschaft vertretenen Position wurde die Kritik verbreitet, dass die erwarteten positiven Effekte durch TTIP bei genauerer Betrachtung nicht sehr hoch ausfallen: Selbst wenn die breit rezipierte Kritik an der Studie unberücksichtigt bleibt ( Raza 2014), können von TTIP im besten Falle verschwindend geringe BIP-Wachstumseffekte von 0,05 Prozent jährlich und einmalig in einem Zeitraum von 10 Jahren erwartet werden. Das musste schließlich selbst der ehemalige Handelskommissar der EK, Karel de Gucht, vor laufender Kamera in einer Fernsehshow der ARD eingestehen ${ }^{3}$. Damit ging der Pro-TTIPFraktion eines ihrer wichtigsten Argumente verloren. Umso glaubwürdiger lässt sich seither die Position vertreten, dass TTIP einigen wenigen Großkonzernen nützt, nicht aber im Interesse aller europäischen Bürger

3 ARD-Monitor, Video. Online: http://www.attac.de/ kampagnen/freihandelsfalle-ttip/hintergrund/studienkritik/ [31.1.2014] 
und Bürgerinnen ist. Um also die Zustimmung für das Abkommen zu stärken, wurde es zunehmend notwendig, in verschiedenen Gremien Stimmung für TTIP (und CETA) zu machen, und die Frage, wem denn nun TTIP tatsächlich zugute kommen würde, erlangte erneut an Brisanz in den Auseinandersetzungen.

In diesen Kontext lässt sich die seit Sommer 2015 forcierte Behauptung, TTIP käme besonders KMU zugute, als politischer Schachzug interpretieren, der zusammen mit anderen Maßnahmen - z.B. dem von der EK veröffentlichten Dokument „The top 10 myths about TTIP“ ( EK 2015e) - darauf abzielt, Kritiker*innen die Argumentationsgrundlagen $\mathrm{zu}$ entziehen und erneut den Versuch startet, TTIP als Projekt im Allgemeininteresse darzustellen. Die Verschiebung der Debatte über die Gewinner*innen von TTIP hin zu KMU ist dabei besonders interessant, da KMU eine weitere potenzielle Konfliktachse im Kampf um TTIP darstellen und eine Bruchlinie im „hegemonialen Block“ sichtbar werden lässt.

In diesem Beitrag wird die Debatte über die Auswirkungen von TTIP auf KMU aus hegemonietheoretischer Perspektive interpretiert und analysiert. Nach einer kurzen Darstellung des verwendeten Hegemoniebegriffs und der analytischen Herangehensweise wird die Darstellung der EK kritisch betrachtet werden. In einem zweiten Schritt werden aktuelle Daten zum globalen Handel mit Daten zu Unternehmenscharakteristika kombiniert und aufgearbeitet, um die makroökonomischen Chancen für KMU im globalen Handel anhand empirischer Daten zu diskutieren. Abschließend wird anhand einer kurzen Fallanalyse (aus Österreich) gezeigt werden, dass TTIP jenseits von makroökonomischen Wachstumschancen das Interesse großer, global agierender Unternehmen widerspiegelt und deren Position gegenüber gewählten Regierungen stärkt.

\section{Hegemonietheorie}

Der Begriff der „Hegemonie“ geht auf den Staatstheoretiker Antonio Gramsci zurück ${ }^{4}$. Mit seiner originellen Analyse erweiterte dieser grundlegend den „traditionellen" Staatsbegriff, worunter in erster Linie ein polizeilicher, militärischer und administrativer Apparat verstanden wurde, dessen Funktion die Vermittlung

4 Eine gute Einführung in das Staatsverständnis nach Gramsci bietet das Buch „Hegemonie gepanzert mit Zwang“ (Buckel, Fischer-Lescano 2007). gesellschaftlicher Widersprüche ist (Demirovic 2007: 21ff). Nach Gramsci ist ein solches Verständnis des Staates, als Instrument der herrschenden Klasse, jedoch nicht ausreichend, um die kapitalistischen Staaten (des Nordwestens Anfang des 20. Jahrhunderts) zu beschreiben. Herrschaft basiert nicht ausschließlich auf dem Gewaltmonopol eines bürokratischen Staatsapparates mit der Funktion, die Interessen der herrschenden Klasse aufrecht zu erhalten. Vielmehr bedarf es, um nachhaltig Herrschaft zu stabilisieren, eines „Konsenses“ in der „Zivilgesellschaft“. Das heißt, es bedarf der Zustimmung zur herrschenden Ideologie, welche in Bildungseinrichtungen, Kirchen, (Massen-) Medien, Gewerkschaften, Nichtregierungsorganisationen (NGOs), Vereinen und dergleichen produziert und reproduziert wird. Staat wird dann selbst als Gesellschaft gedacht, nämlich als der (operative) politische Bereich, während die Zivilgesellschaft als Ort der Erzeugung, Festigung und Aufrechterhaltung von Herrschaft verstanden wird.

„In dem Sinne könnte man sagen, daß Staat = politische Gesellschaft + Zivilgesellschaft, das heißt, Hegemonie, gepanzert mit Zwang“. (Gramsci 1991:83 nach Demirovic 2007:24)

Gramsci erweiterte jedoch nicht nur die für eine Herrschaftsanalyse notwendigen Bereiche des Staates um die Zivilgesellschaft, sondern er strich auch die Funktion der Zustimmung für die Stabilisierung von Herrschaft heraus. Der Vermittlung von „Ideologie“ in der Zivilgesellschaft wird somit eine wesentliche Rolle in der Ausübung von Herrschaft zugeschrieben.

Der Fokus auf die Vermittlung von Ideologie als Bestandteil von Herrschaft ermöglicht es in weiterer Folge zu argumentieren, dass Herrschaft nicht auf ein „Klassenbewusstsein“ zurückgeführt werden muss, welches sich essentialistisch, aus der Funktion und den verknüpften Erfahrungen, aus der Position im Produktionsprozess ableiten lässt - sondern selbst, politisch erzeugt wird.

Im Gegensatz zur diskursorientierten Auslegung von Hegemonie bei Laclau und Mouffe (2006) bleiben bei Gramsci die Klassenverhältnisse als Basis der kapitalistischen Gesellschaft jedoch bestehen. Das „eigentliche“ Ziel des kapitalistischen Staates bleibt die Aufrechterhaltung der Herrschaft der Bourgeoisie über den Produktionsapparat. Kompromisse werden zwar von Zeit zu Zeit notwendig, um kapitalistische, auf Eigentum und Akkumulation gebaute Gesellschaftsformen und die damit einhergehenden Privilegien der herrschenden Gruppen aufrecht zu erhalten. An den 
Grundsätzen der bürgerlichen Gesellschaft wird jedoch nicht gerüttelt. Soweit ist Staat

„ein fließendes Kräftegleichgewicht, das jedoch asymmetrisch bleibt, weil er immer die Partei derjenigen ist, die den Produktionsapparat organisieren; er ist Prozess und Ergebnis der Verallgemeinerung und der Ausarbeitung von Kompromissen als Grundlage des Konsenses. Bei den den Staat tragenden Kräften liegt die Initiative, sie organisieren im und durch den Staat den gesellschaftlichen Kollektivwillen." (Demirovic 2007:33)

Bei diesen staatstragenden Kräften handelt es sich jedoch nicht um eine einheitliche Gruppe, die sich durch ein genuines politisches Bewusstsein auszeichnet und dieselben Interessen verfolgt. Klassen werden erst über den Staat als politische Kräfte konstituiert (Poulantzas 1965:866-869 nach Jessop 2007:50). Denn die Bourgeoisie selbst ist in unterschiedliche Klassenfraktionen gespalten: (grob) in Monopolkapital und nichtmonopolistisches Kapital ( Poulantzas 2002:158f.). Zwischen diesen (archetypischen) Fraktionen des Blocks an der Macht behält der Nationalstaat immer eine relative Autonomie, „[...] um so die Organisierung des Allgemeininteresses der Bourgeoisie unter der Hegemonie einer ihrer Fraktionen sicherzustellen“ (Poulantzas 2002:159). Die Aufgabe des Staates ist es also immer auch, den Block an der Macht zu organisieren und $\mathrm{zu}$ vereinheitlichen. Somit bedarf Herrschaft auch einer Vereinheitlichung, eines Konsensus unter den verschiedenen Kapitalfraktionen. Die Tendenzen des Kapitalismus zur Monopolbildung müssen von einer relativ autonomen Staatlichkeit vermittelt und organisiert werden.

Zwar sind Gramscis und Poulantzas Konzepte der Hegemonie im Nationalstaat verankert, allerdings spricht nichts dagegen, die Konzepte für die Analysen der Transnationalisierung von Staatlichkeit heranzuziehen (Bieling 2007) und somit für die Analyse von TTIP dienlich zu machen. Wird der Prozess der Transnationalisierung und dabei der Prozess der Verschiebung von handelspolitischen Kompetenzen von der nationalstaatlichen auf die europäische Ebene in den Blick genommen, so lässt sich dieser Prozess selbst als Teilaspekt hegemonialer Verstaatlichung fassen. Mit Verweis auf das analytische Konzept des „Neuen Konstitutionalismus“ von Stephen Gill (1995) lässt sich TTIP dann als eine spezielle Form der markt- und wettbewerbszentrierten (Neu-)Konstitutionalisierung von Staatlichkeit auf transnationaler Ebene beschreiben und analysieren. Die Konstitutionalisierung der Weltwirtschaft wird dabei als ein Prozess hegemonialer Verstaatlichung betrachtet (Bieling 2007:144).
Dieser Prozess der (ökonomischen) Neukonstitutionalisierung nationaler Kompetenzen auf inter- und transnationaler Ebene geht mit einer Verschiebung der Kräfteverhältnisse zugunsten inter- und transnationaler Kapitalfraktionen einher. Bereits Poulantzas bemerkte, dass sich die Fraktionierung zwischen Monopol und nicht monopolistischem Kapital im Prozess der Internationalisierung des Staates verstärkt (Poulantzas 2002:158f.). Dabei bieten einerseits unterschiedliche Foren der politischen Auseinandersetzungen strategische Vorteile für gewisse soziale Gruppen. So bietet die starke Exekutivlastigkeit der Europäischen Union einen strategischen Vorteil für gut organisierte und machtvolle Lobbygruppen (Becker et al. 2015:12f.). Die EU zeichnet sich durch eine „strategische Selektivität“ (Jessop 2002:40) zugunsten transnationaler Unternehmens- und Finanzkapital-Fraktionen aus ( Becker et al. 2015:13). Andererseits verändert die „Reskalierung“ der Räumlichkeit einen wichtigen Aspekt der strategischen Gestaltung struktureller Machtverhältnisse. Beispielsweise untergraben politische Bemühungen zur künstlichen Verschärfung globaler Wettbewerbsverhältnisse kollektive, solidarische Praktiken wie Kollektivverträge, indem Arbeiter*innen in Ländern mit hohen Sozialstandards mit Arbeiter*innen in Ländern mit niedrigen Sozialstandards in Konkurrenz gesetzt werden. Als Konsequenz wird Druck auf Arbeitseinkommen erzeugt und Lohnsenkungen lassen sich leichter umsetzen. So bemerkte beispielsweise Immanuel Wallerstein (1979:292), dass partikulare soziale Gruppen ihre Vorteile modifizieren können, indem sie die Staatsgrenzen modifizieren. Möglichkeiten der wirtschaftspolitischen Steuerung (Sozialpolitik, Umverteilung, Regionalentwicklung ...) können schließlich durch die Neukonstitutionalisierung von Rechtsstaatlichkeit (z.B. durch Handelsverträge, Schiedsgerichte, Regulierungsabkommen ...) auf nationalstaatlicher Ebene eingeschränkt werden. Gleichzeitig erweitern sich aber auch die staatlichen Regulationsmöglichkeiten, indem die räumliche Begrenztheit nationalstaatlicher Herrschaft partiell überwunden wird. Ermöglicht durch asymmetrische ökonomische Interdependenzen zwischen Staaten lassen sich (handels-)politische Forderungen in Foren wie der World Trade Organization (WTO), aber auch durch bilaterale Abkommen wie TTIP über die Grenzen nationalstaatlicher Herrschaftsräume hinaus umsetzen. Gewisse Staaten werden somit zu einer wichtigen Arena und Basis für die Etablierung von Machtverhältnissen auf globaler Ebene. „Zumindest für die entwickelten kapitalistischen Staaten - allen voran 
die USA und die Mitgliedsstaaten der Europäischen Union - eröffnet die institutionell-vertragsrechtliche Absicherung der internationalen Wirtschaftsbeziehungen die Möglichkeit, ihre wirtschaftspolitische Liberalisierungsagenda global zu verallgemeinern“ (Bieling 2007:152). Unter diesem Aspekt geht es bei CETA und TTIP also um weitaus mehr als um transatlantische Abkommen, da die Verträge als Blaupause für bilaterale Handelspolitik in der Zukunft verwendet werden können.

Da der Prozess der Transnationalisierung von Staatlichkeit Fragmentierungen entlang nationaler Grenzen und Klassenunterschiede aufweist, bedarf es umso mehr einer umfassenden materiellen und ideologischen Einbettung verschiedener sozialer Kräfte, um einen Konsens für die Teilaspekte der transnationalen Verstaatlichung zu erzielen. Eine zentrale Rolle dabei spielen einflussreiche nationale Politiker*innen, transnationale Allianzen und supranationale Institutionen, welche in Gramscis Begrifflichkeit das Erscheinen eines transnationalen hegemonialen Blocks sozialer Kräfte repräsentieren (Bieling/Jäger/Ryner 2015:6). Dieser Block vermag es dann hegemonial zu werden, wenn es gelingt, das Spiel zum Vorteil der dominanten Gruppen zu verändern, während die untergeordneten Gruppen diese Veränderung als vorteilhaft für sich selbst interpretieren ( ebd.). In anderen Worten, wenn es gelingt, den Prozess der Transnationalisierung im Interesse der transnational agierenden Gruppen $\mathrm{zu}$ gestalten und dieses Partikularinteresse als Gemeininteresse zu verallgemeinern.

Das Ziel von Herrschaft kann dann als Versuch, politische und ökonomische Strukturen im Sinne der dominanten Gruppe zu verändern oder zu bewahren und global auszuweiten, verstanden werden. Ein entsprechender Konsens, bei dem die dominante herrschende Fraktion ihr spezifisches Eigeninteresse glaubwürdig als Gemeininteresse etabliert, ermöglicht es dabei, Herrschaft mit einem geringen Ausmaß an Zwangsmomenten auszuüben. Dabei bedarf es sowohl einer Vereinheitlichung fragmentierter Klassen(interessen) als auch der Zustimmung in der Zivilgesellschaft.

TTIP lässt sich somit als ein Teilaspekt der hegemonialen transnationalen Verstaatlichung begreifen. Um Hegemonie möglichst ohne Zwang auszuüben, muss der „transnationale Block“ einen möglichst breiten Konsens erzeugen. Dieser ist von sozialen Kräften entlang verschiedener Dimensionen umkämpft. Die Verallgemeinerung von Partikularinteressen ebenso wie die Vereinheitlichung von Klassenfraktionen, aber auch das Etablieren eines Bewusstseins als politische Klasse oder Gruppe gehören zu den zentralen Dimensionen dieses politischen Kampfes, obgleich die Art und Weise, wie CETA umgesetzt wird, darauf hinweist, dass dies ohne Zwang nicht mehr möglich ist.

Die Aussage „TTIP kommt besonders KMU zugute" kann nun als ein Versuch der Verallgemeinerung von Partikularinteressen im Zuge der Neukonstitutionalisierung ökonomischer Rahmenbedingungen begriffen werden, mit dem impliziten Versuch der Vereinheitlichung von Kapitalfraktionen und der Konsequenz, dass sich eine weitere Bruchlinie zwischen TTIP befürwortenden und ablehnenden politischen Gruppen aufgetan hat: nämlich der Interessenskonflikt zwischen den kleinen, lokal, national und regional orientierten sowie den großen, transnational orientierten Unternehmen.

\section{Analytische Überlegungen}

Um die Auswirkungen von TTIP auf KMU unter dem Aspekt von Herrschaftsverhältnissen analysieren zu können, werden im Folgenden unterschiedliche Perspektiven $\mathrm{zu}$ demselben Thema eingenommen und dabei hegemonietheoretisch interpretiert. Erstens wird ein kritischer Blick auf die Darstellung und Kommunikationsstrategie der EK geworfen, zweitens auf die Inhalte der Debatte und auf die Plausibilität der propagierten Auswirkungen. Dabei wird bewusst eine TTIP-skeptische Position eingenommen und eine alternative Darstellung und Interpretation möglicher Auswirkungen anhand selbst gesammelter Daten vorgenommen. Aus hegemonietheoretischer Perspektive wird dabei die Debatte unter dem Aspekt der politischen Möglichkeit zur Veränderung ökonomischer Rahmenbedingungen mit dem Ziel der möglichst vorteilhaften (Re-)Produktion der materiellen Basis analysiert.

Durch den Blick auf die Debatte, in diesem Fall auf die Kommunikationsstrategie bzw. die Darstellung der EK, soll gezeigt werden, mit welchen Mitteln versucht wird, TTIP als besonders vorteilhaftes Abkommen für KMU darzustellen. Der Diskurs wird dabei als politisch strategisches Instrument verstanden, um, im Rahmen des festgelegten Prozedere, des „Better Regulation Programs“ (BRP), Zustimmung für eine politische Maßnahme (in diesem Fall TTIP) zu erlangen. Herrschaftsverhältnisse werden dabei verschleiert, ein Konsens wird angestrebt. 
Ein Blick auf „die Daten“ sowie auf die bekannten Inhalte von TTIP hilft dann die erwarteten Effekte des umkämpften Regulierungsabkommens einzuschätzen, liefert bewusst eine Gegendarstellung zu der kritisierten Darstellung der EK und gibt Anhaltspunkte, um die Gewinntragenden des Abkommens zu identifizieren.

Als theoretischer Rahmen und Orientierungspunkt zur Verortung der Analyse fungieren die bereits dargestellten hegemonietheoretischen Überlegungen.

\section{KMU und TTIP - die Darstellung der EK}

Um geplante Innovationen möglichst reibungslos umzusetzen, folgt die EK einem eigens vorgegebenen Prozedere, dem sogenannten „Better Regulation Program“ (BRP). Dieses ist darauf ausgelegt, die notwendige Zustimmung für geplante Initiativen $\mathrm{zu}$ sichern, bevor die Entwürfe der EK dem Europäischen Parlament (EP) zur Abstimmung vorlegt werden (EK 2015c). Das BRP lässt sich also als eine zentrale konsensstiftende Maßnahme der EK verstehen und bietet interessante Ansatzpunkte für die weitere Analyse. Dabei werden zwei Punkte des BRP besonders in den Fokus gerückt. Erstens ist hier die Rolle der eigens angefertigten Folgenabschätzung zu nennen, die ein zentrales Element des BRP darstellt. Dabei sollen durch wissenschaftliche Studien mögliche ökologische, soziale und ökonomische Folgen eruiert werden, bevor über die Sinnhaftigkeit eines Abkommens entschieden wird. Zweitens sieht das BRP vor, die geplanten Initiativen durch das Miteinbeziehen von Interessensvertreter*innen $\mathrm{zu}$ stützen. Durch die Konsultation von Interessensgruppen, die von der geplanten Initiative betroffen sind, sowie durch das Durchführen von Umfragen, welche unter anderem in die Studien integriert werden, soll ein entsprechender Rückhalt erzeugt werden.

Prinzipiell lässt dieses Prozedere Spielraum für eine begrüßenswerte politische Praxis. Allerdings ist gesellschaftliches Handeln von Machtstrukturen durchzogen (Bhaskar 2008), weshalb ein solcher Prozess immer auch die zugrundeliegenden Hierarchien widerspiegelt. So steht beispielsweise eine Nachhaltigkeitsprüfung von TTIP nach wie vor aus ${ }^{5}$, obgleich schon ein Großteil der Verhandlungen geführt wurde. Ergebnisse einer Nachhaltigkeitsprüfung im Nachhinein, sofern sie überhaupt durchgeführt werden, wären dann eher Kosmetik, als dass sie noch tatsächlich Einfluss auf die

5 Link zu den Assessment Studien: Online: http:// www.trade-sia.com/ttip/ [Oktober 2016]
Konstitutionalisierung der Handelsarchitektur Einfluss nehmen könnten.

Seit Beginn der TTIP-Verhandlungen lässt sich beobachten, wie sich Konzerne auf beiden Seiten des Atlantik organisiert und für TTIP stark gemacht haben ${ }^{6}$. Corporate Europe Observatory (CEO) zeigt in einer Statistik, welche Interessensvertretungen am häufigsten bezüglich TTIP konsultiert wurden, nämlich international tätige Konzerne, auf die insgesamt 93 Prozent der gesamten Konsultationsdauer fallen (CEO 2013). Entgegen der aktuellen Darstellung der EK, wonach bei TTIP Interessensgruppen der Zivilgesellschaft entscheidend in den Gestaltungsprozess des Abkommens einbezogen würden, hat sich daran nicht viel geändert, außer, dass nun auch KMU kritisch gegen TTIP auftreten (CEO 2015). In Hinblick auf das BRP wird somit schon hier deutlich, von wem und für wen TTIP konzipiert ist.

Um dennoch Konsens für die Initiative zu erlangen, ist es also umso wichtiger, die zugrunde liegenden Interessen $\mathrm{zu}$ verallgemeinern und $\mathrm{zu}$ etablieren, dass Vorteile von TTIP einem möglichst großen Zielpublikum zugutekommen werden. Eben das scheint der argumentative Schwenk hin zu den vermeintlichen Vorteilen von TTIP für KMU zu sein. Wie schon bei dem blassen Versprechen von mehr Jobs und Wachstum, wird dabei erneut auf die Autorität „wissenschaftlicher" Studien gesetzt. So veröffentlichte die EK parallel zum Start der TTIP-KMU-Kampagne im Sommer 2015 einen Report, in dem die Vorteile von TTIP für KMU dargelegt werden (EK 2015b). Ein genauerer Blick auf die Genesis der Studie zeigt dabei exemplarisch, wie dieses Mittel als PR-Maßnahme eingesetzt wird bzw. wie versucht wird, Konsens zu erzeugen, indem Partikularinteressen verallgemeinert werden.

Vorbereitet wurde der Bericht in der „Chief Economist Note" der EK, verfasst von Cernat et al. (2014). Dabei nehmen die Verfasser*innen Bezug auf ein bereits im Jahr 2008, noch vor dem offiziellen Beginn der Verhandlungen zu TTIP, veröffentlichtes Paper von Mayer und Ottaviano (2008). Die beiden Autoren analysieren in dem Paper die Performance von Unternehmen unterschiedlicher Größe im internationalen Handel. Dabei kommen sie zu dem Ergebnis, dass einige wenige Unternehmen den Großteil der aggregierten Exporte erzeugen ( Mayer/Ottaviano 2008). Diese zeichnen sich

6 Dabei wird unter anderem dem Transatlantic Business Council (TABC) eine wichtige Rolle zugeschrieben. (Elsner 2015b) 
durch ihre Größe und durch ein entsprechend breit diversifiziertes Produktangebot aus (Mayer/Ottaviano 2008:17). Mit anderen Worten, es handelt sich im internationalen Handel um stark monopolisierten Wettbewerb. Im Kontext des wachsenden Widerstandes gegen TTIP ist es für Befürworter*innen jedoch wenig hilfreich, auf die Problematik hinzuweisen, dass der internationale Handel einigen wenigen Unternehmen zugutekommt. Stattdessen wird versucht, die Chancen für KMU in den Vordergrund zu stellen. Die empirischen Ergebnisse von Mayer und Ottaviano werden dabei uminterpretiert und in ein anderes Licht gestellt:

"One of the conclusions of this emerging literature is that international trade is a world of 'happy few' exporters. Based on a sub-set of EU Member State firm-level data, Mayer and Ottaviano (2007) found that a handful of firms accounts for a disproportionate share of aggregate exports. While this conclusion is true in relative terms and supported by many other subsequent analyses, this may not be true in absolute terms." (Cernat et al. 2014: 3)

Cernat et al. argumentieren also, dass KMU zwar nur eine sehr geringe Rolle in Relation $\mathrm{zu}$ großen Unternehmen spielen, verweisen jedoch gleichzeitig darauf, dass KMU in absoluten Zahlen eine besonders wichtige Rolle im internationalen Handel spielen. Da 81 Prozent aller im EU-Export tätigen Unternehmen KMU sind (in absoluten Zahlen), kommen Cernat et al. zu dem Schluss, dass diese Unternehmen auch besonders von handelserleichternden Maßnahmen profitieren würden. Mit diesem Kunstgriff wird also eine alltagstaugliche Argumentation erzeugt, die eine Rationalität für die vermeintlichen Vorteile von TTIP für KMU schaffen soll.

Die Schlussfolgerung übernimmt die EK schließlich in dem bereits erwähnten Report (EK 2015b), der 2015, parallel zum Beginn der TTIP-KMU-Kampagne, publiziert wurde. Der Report, welcher erneut unter Mitwirkung des EK-nahen Think Tank ECORYS verfasst wurde, entspricht genau der Argumentation von Lucian Cernat. Der wesentliche Unterschied ist, dass neben aktuelleren ökonomischen Handelsdaten das Ergebnis einer quantitativen Umfrage über die Bedeutung von Nicht-tarifären Handelsbarrieren (NTB) für KMU integriert wurde. Bei dieser wurden nach Angaben der Autor*innen verschiedene KMU innerhalb der EU gefragt, was für sie die größten Kosten und Hürden beim Warenhandel mit den USA seien. Nicht überraschend ist das Ergebnis, wonach die Beseitigung von NTB eine wichtige handelserleichternde Maßnahme für die befragten Unternehmen darstelle. Das Ergebnis scheint jedoch nicht sehr robust zu sein, denn die zugrunde liegende Stichprobe spiegelt nicht die tatsächliche Verteilung der Unternehmen wider (Elsner 2015a). Von den 869 befragten Unternehmen sind 279 (das sind 32,1 Prozent) Kleinstunternehmen, 226 (entspricht 26,o Prozent) Kleinunternehmen, 222 (also 25,5 Prozent) sind mittelständische Unternehmen und 142 (d.h. 16,3 Prozent) zählen zu den Großunternehmen. Die Verteilung der Gesamtheit aller europäischen Unternehmen sieht jedoch anders aus. Kleinstunternehmen machen 92,1 Prozent, Kleinunternehmen 6,6 Prozent und mittelständische Unternehmen 0,2 Prozent aus (ebd.).

Ein zentraler Aspekt der Argumentationsstrategie der EK ist, dass Liberalisierungsbemühungen in einer Welt, in der es kaum noch tarifäre Zölle gibt, auf die Beseitigung von NTBs verschoben werden. Hierbei handelt es sich nicht um eine politische Ausrichtung, die sich explizit bei TTIP beobachten lässt, sondern um eine generelle Verschiebung des handelspolitischen Fokus der EK, was beispielsweise auch an der „neuen Handelspolitik ${ }^{\text {“7 }}$ der EK ablesbar ist. Die dabei stattfindende inhaltliche Erweiterung der Handelspolitik auf sogenannten NTBs und damit auf Themenbereiche, welche weit über die Reduktion von Tarifen hinausgehen, werden unten behandelt.

$\mathrm{Zu}$ bemerken bleibt, dass diese inhaltlichen Erweiterungen der Handelspolitik in die altbekannte und in den letzten Jahren „bewährte“ Argumentation der Wettbewerbsfähigkeit eingebettet werden. So wird auch in der Debatte über die Auswirkung von TTIP auf KMU argumentiert, es käme zu einer Stärkung des Wettbewerbes über internationalen Handel. Das ist jedoch nur ein Teil der ökonomischen Effekte in der handelstheoretischen Fundierung nach Paul Krugman (2009). Theoretisch werden dabei zwei mögliche Auswirkungen für den internationalen Handel unterschieden: zunehmende Monopolisierung wegen der höheren Skalenerträge großer Unternehmen, sowie ein Anstieg der Konkurrenz wegen dem Wunsch nach Vielfalt seitens der Konsument ${ }^{*}$ innen („love of variety“). Wie bereits erwähnt, wird in der Argumentation der EK nur der zweite Punkt hervorgehoben. Eine Ausweitung des Wettbewerbes, was als das Ziel von TTIP angeführt wird, führt in dieser Logik zu mehr

7 Die Neue Handelspolitik auf der Homepage der Europäischen Kommission: Online: http://ec.europa.eu/ trade/policy/in-focus/new-trade-strategy/ [Oktober 2016] 
Effizienz, weil unproduktive (kleine) Unternehmen (siehe Mayer/Ottaviano 2008) vom Markt vertrieben werden. Wahrscheinlicher ist es jedoch, dass eine Ausweitung des Konkurrenzdruckes entlang der Produktionskette nach unten, vom Endprodukt über KMUs bis hin zu den Arbeiter*innen, eine Reduktion von deren Preisen erwarten lässt, während die Profite am anderen Ende der Produktionskette steigen. Denn mehr Konkurrenz senkt die Preise unter den Zuliefernden und Arbeiter*innen, während kaum Konkurrenz am oberen Ende der Wertschöpfung vorhanden ist.

Interessanterweise stößt der Versuch, die Vorteile von TTIP für KMU verstärkt in die Debatte zu integrieren, auf den Widerstand derselben. Der Versuch, die Interessen der Handelsliberalisierung für KMU $\mathrm{zu}$ verallgemeinern, scheint nicht $\mathrm{zu}$ funktionieren. Da KMU unter anderem befürchten, dass TTIP zu ihrem Nachteil als Bürger*innen und einzeln als Unternehmer*innen beiträgt (Theurl/Grumiller 2016), haben sich diese unter anderem in einer eigenen Plattform mit dem Namen „KMU gegen TTIP“8 organisiert, um ihre Ablehnung gegen TTIP öffentlich zu kommunizieren. In der Debatte um TTIP scheint somit (unter anderem) der Versuch der Vereinheitlichung der Kapitalfraktionen zu scheitern - nicht zuletzt wegen dem Widerstand und der Mobilisierung von NGOs und teilweise auch Wirtschaftsverbänden.

Im Zuge von TTIP brechen somit die Bruchstellen innerhalb des „hegemonialen Blocks“ weiter auf. Die marktzentrierte, neoliberale Wirtschaftspolitik scheitert zunehmend an ihrer eigenen Widersprüchlichkeit, denn sie ist nicht mehr in der Lage, die Akkumulationsmöglichkeiten für alle Kapitalfraktionen gleichermaßen zu sichern (Apeldoorn 2014). Der Block an der Macht vermag es immer weniger, den Glauben aufrecht zu erhalten, dass die „dominierten“ Gruppen von der Politik der Herrschenden profitieren.

\section{Die Rolle von europäischen KMU im globalen Handel}

Im Folgenden soll eine Gegendarstellung vorgenommen werden und die Reichweite der Handelsabkommen, weit über Zollreduktionen hinaus, thematisiert werden.

KMU spielen in mehrfacher Hinsicht eine wichtige politische und ökonomische Rolle in Europa

8 Link zur Homepage: http://kmu-gegen-ttip.at [10. Oktober 2016]
(Grumiller/Theurl 2015). 2011 gab es mehr als 20,7 Millionen KMU in der EU, das sind über 98 Prozent aller Unternehmen. Sie produzieren 58 Prozent der Bruttowertschöpfung, beschäftigen 67 Prozent der Arbeitnehmer*innen (EK 2015d) und fungieren im nicht finanziellen Wirtschaftssektor als Wachstumsmotor der EU (Schmiemann 2009). Doch während KMU eine zentrale Rolle in der europäischen Ökonomie spielen, trifft das weder auf den EU-Außenhandel noch auf den Handel zwischen EU und USA zu.

Wie schon erwähnt, wird bei der Debatte um Möglichkeiten für KMU auf globalen Märkten von den Befürworter*innen ein einseitiges Bild gezeigt, indem ausschließlich die Anzahl jener KMU, die im EU-Außenhandel tätig sind, genannt wird. Demnach waren im Jahr 2011 beachtliche 81 Prozent der Unternehmen auf globalen Märkten als KMU klassifiziert. Damit wird suggeriert, dass vor allem KMU von handelserleichternden Maßnahmen profitieren würden. Allerdings lässt sich daraus nicht schließen, dass Handelserleichterungen generell im Sinne aller KMU wären. Insgesamt spielt der globale Handel für KMU keine besondere Rolle. Von den 2011 registrierten 20,7 Millionen KMU (EK 2015d) waren nämlich nur 3 Prozent der europäischen KMU auf globalen Märkten tätig. Betrachtet man die Zahl der europäischen KMU, die Handelsbeziehungen mit den USA pflegen, dann zeigt sich, dass auch hier 2012 weniger als 1 Prozent aller KMU Handelsbeziehungen mit den USA hatten (Grumiller/Theurl 2015). Entgegen der aktuellen Darstellung der TTIP-Befürworter ${ }^{\star}$ innen würde das geplante Abkommen nicht generell KMU zugutekommen.

Darüber hinaus ist es ebenfalls wichtig, darauf hinzuweisen, dass selbst international orientierte KMU eine untergeordnete Rolle auf globalen Märkten spielen. Obwohl 81 Prozent aller Unternehmen auf globalen Märkten als KMU klassifiziert sind, tragen diese gerade einmal 34 Prozent zum aggregierten Export bei. Dem gegenüber stehen große Unternehmen mit mehr als 250 Angestellten. Mengenmäßig machen diese gerade einmal 3 Prozent der europäischen Unternehmen auf globalen Märkten aus, erwirtschafteten jedoch 53 Prozent und somit den Großteil des aggregierten Exports ${ }^{9}$ (siehe Tabelle 1).

9 Der Rest auf 100 Prozent fällt auf Unternehmen, die keine Angaben über ihre Größe machten. 


\begin{tabular}{|c|c|c|c|c|c|c|c|}
\hline & $\begin{array}{c}\text { Kleinst } \\
(1-9)\end{array}$ & $\begin{array}{c}\text { Klein } \\
(10-49)\end{array}$ & $\begin{array}{c}\text { Mittel } \\
(50-249)\end{array}$ & $\begin{array}{c}\text { Groß } \\
(250+)\end{array}$ & $\begin{array}{c}\text { Keine } \\
\text { Angaben }\end{array}$ & $\begin{array}{c}\text { Summe } \\
\text { KMU }\end{array}$ & $\begin{array}{c}\text { Summe } \\
\text { exportierende } \\
\text { Unternehmen }\end{array}$ \\
\hline Exportierende Unternehmen & 352.900 & 187.402 & 73.551 & 23.319 & 121.556 & 613.853 & 759.849 \\
\hline Aggregierte Exporte (Mio. EUR) & 122.303 & 143.388 & 254.454 & 816.300 & 210.055 & 520.145 & 1.546 .499 \\
\hline Prozent der Unternehmen & $46 \%$ & $25 \%$ & $10 \%$ & $3 \%$ & $16 \%$ & $81 \%$ & $100 \%$ \\
\hline Prozent aggregierte Exporte (Mio EUR) & $8 \%$ & $9 \%$ & $16 \%$ & $53 \%$ & $14 \%$ & $34 \%$ & $100 \%$ \\
\hline
\end{tabular}

Quelle: eigene Darstellung, EuroStat TEC Datenbank

Tabelle 2: Export EU - USA, 2011

\begin{tabular}{|c|c|c|c|c|c|}
\hline & $\begin{array}{l}\text { exportierende } \\
\text { Unternehmen }\end{array}$ & Prozent & $\begin{array}{l}\text { Aggregierte Exporte } \\
\quad \text { (in Mio USD) }\end{array}$ & Prozent & $\begin{array}{c}\text { Aggregierte Exporte/Un- } \\
\text { ternehmen }\end{array}$ \\
\hline Gesamt & 183.960 & $100 \%$ & 1.945 .307 & $100 \%$ & 10,57 \\
\hline Kleinst & 120.109 & $65 \%$ & 210.938 & $11 \%$ & 1,76 \\
\hline Klein & 37.689 & $20 \%$ & 124.895 & $6 \%$ & 3,31 \\
\hline Mittel & 17.724 & $10 \%$ & 185.112 & $10 \%$ & 10,44 \\
\hline Groß & 8.438 & $5 \%$ & 1.424 .361 & $73 \%$ & 168,80 \\
\hline
\end{tabular}

Quelle: Eigene Darstellung, OECD TEC Datenbank

Dasselbe gilt für den Handel zwischen den USA und Europa. Die Daten zeigen, dass auch der amerikanische Markt für große Unternehmen lukrativer ist als für kleine (Tabelle 2). KMU machen erneut den Großteil der Handel treibenden Unternehmen aus und tragen auch in diesem Fall nur sehr wenig zum aggregierten Export bei. Teilt man rechnerisch die aggregierten Exporte auf die Anzahl der Unternehmen auf, so lässt sich das numerisch veranschaulichen. Während auf dem Exportmarkt von der EU in die USA durchschnittlich Güter im Wert von 10,57 Millionen USD verkauft werden, fällt auf Kleinst-Unternehmen ein durchschnittlicher aggregierter Güterabsatz in der Höhe 1,76 Millionen USD pro Unternehmen. Auf große Unternehmen fällt demgegenüber ein aggregierter Güterabsatz im Wert von 168,8 Millionen USD. Obwohl diese Werte relativ wenig Informationen über die ökonomische Rolle und Performance der Unternehmen liefern, veranschaulichen sie, dass große Unternehmen einen bemerkenswerten Vorteil aufgrund ihres Kapitalvolumens besitzen. Das bedeutet, dass sie im Gegensatz zu KMU leichter zu Krediten kommen, sich eher Preiskriege leisten können, sich besser gegen Wechselkursschwankungen absichern können, leichter den Standort wechseln, um niedrigere Lohnstückkosten ausnutzen zu können, und sich eher Verhandlungen vor internationalen Schiedsgerichten leisten können, um nur einige Beispiele zu nennen.

Es lässt sich also festhalten, dass große Unternehmen tendenziell mehr von globalen Märkten profitieren als kleine Unternehmen und somit auch ein größeres Interesse haben, auf diesen tätig zu sein. Da die aktuellen Zahlen eine Situation abbilden, in der kaum noch Handelsbarrieren existieren, ist es nicht nachvollziehbar, wie ein weiterer Abbau bestehender Handelsregeln besonders KMU zugutekommen soll.

Die Antwort, die in der Debatte um die neue Generation von Handelsverträgen meist genannt wird, lautet nicht-tarifäre Barrieren (NTB). Diese sollen beispielsweise für den Großteil der ökonomischen Effekte verantwortlich sein, die durch TTIP in Aussicht gestellt werden. Jedoch bleiben sie gleichzeitig vage definiert und umfassen all das, was Kosten im Handel verursachen kann. Eine Nebenkonsequenz davon ist, dass der Inhalt der Verhandlungen somit noch stärker von dem Einfluss verschiedener Kräfteverhältnisse abhängig wird. Denn, wie bereits erwähnt, es werden Interessensvertretungen von großen Unternehmen besonders stark in die Gestaltung des tatsächlichen Inhalts von TTIP mit einbezogen (Corporate Europe Watch 2013; Corporate Europe Watch 2015).

Wie bei den bereits bekannten Verhandlungstexten ${ }^{10}$ in TTIP befürchtet wird, führt die Reduktion von

10 Die „Leaks“ der Verhandlungstexte finden sich hier: https://ttip-leaks.org/; eine Analyse von Greenpeace (2016) kommt unter anderem zum Ergebnis, dass Umweltstandards aufgelockert werden. Online: http://www.greenpeace.org/euunit/Global/eu-unit/reports-briefings/2016/TTIP\%2oleaks\%20 analysis.pdf [Oktober 2016] 
Theurl: Die Rolle von Klein- und Mittelunternehmen im politischen Kampf um TTIP. Eine hegemonietheoretische Betrachtung

Tabelle 3: Import EU - USA, 2011

\begin{tabular}{lccccc}
\hline & $\begin{array}{c}\text { exportierende } \\
\text { Unternehmen }\end{array}$ & Prozent & $\begin{array}{c}\text { Aggregierte Exporte (in } \\
\text { Mio USD) }\end{array}$ & $\begin{array}{c}\text { Prozent } \\
\text { Aggregierte Importe/Un- } \\
\text { ternehmen }\end{array}$ \\
\hline Gesamt & 292.533 & $100 \%$ & 1.319 .942 & $100 \%$ & 4,51 \\
Kleinst & 192.239 & $66 \%$ & 187.996 & $14 \%$ & 0,98 \\
Klein & 62.010 & $21 \%$ & 81.954 & $6 \%$ & 1,32 \\
Mittel & 27.274 & $9 \%$ & 120.455 & $9 \%$ & 4,42 \\
Groß & 11.010 & $4 \%$ & 929.537 & $70 \%$ & 84,43 \\
\hline
\end{tabular}

Quelle: Eigene Darstellung, OECD TEC Datenbank

NTB unter anderem zur Erosion bestehender qualitätssichernder Standards (Beck/Scherrer 2014). Das birgt besonders für KMU eine große Gefahr, da diese meist Produkte hoher Qualität produzieren und deshalb entsprechende Preise erzielen müssen, um kostendeckend produzieren zu können. Jedoch sind die Standards in den USA in einigen Bereichen niedriger als jene in Europa. Somit könnte die „Harmonisierung“ der Standards, also ein Hinunterrevidieren höherer Standards, dazu führen, dass KMU durch steigenden Wettbewerb einem höheren Preisdruck und somit Druck auf die Arbeitslöhne und auf die Qualität der Produkte ausgeliefert sind.

Wenn man sich nun die Importzahlen anschaut, sieht man, dass US-amerikanische KMU weitaus weniger in die EU exportieren als umgekehrt (Tabelle 3). Das lässt sich unter anderem darauf zurückführen, dass Produkte in der EU schärferen Qualitätsregeln und Verordnungen entsprechen als in den USA. Eine Abschaffung dieser Regeln würde somit den Wettbewerbsdruck auf KMU in Europa erhöhen und könnte diese möglicherweise vom Markt verdrängen.

Befürworter*innen von TTIP argumentieren jedoch oft, dass Qualitätsstandards und andere von der Öffentlichkeit als problematisch erachtete Regeln sowieso bestehen blieben. Allerdings müssten dann auch die bereits geringen Wachstumseffekte, die durch TTIP in Aussicht gestellt werden, entsprechend nach unten revidiert werden.

Dass die von TTIP erwarteten makroökonomischen Effekte kaum die möglichen Gefahren ausgleichen, die mit dem geplanten Abkommen einhergehen, ist nichts Neues (Raza 2014). Hingegen lässt sich die Behauptung, TTIP käme besonders stark KMU zugute, empirisch nicht belegen. Es ist unklar, ob nicht das Gegenteil der Fall ist (Grumiller/Theurl 2015). Der Fokus auf die vermeintlichen Vorteile von TTIP für KMU sollte deshalb als bewusste strategische Behauptung verstanden werden, die darauf abzielt, einen Konsens für TTIP zu generieren.

\section{Mehr als Handelspolitik}

Wie bereits erwähnt, geht es bei den aktuellen bilateralen Handelsabkommen wie TTIP und CETA nicht um die Beseitigung von Zöllen, sondern um eine inhaltliche Erweiterung der Handelspolitik. Dabei wird weitaus mehr diskutiert als handelspolitische Belange, und zwar durch die Delegierten der EU, welche aufgrund der starken Exekutivlastigkeit offen für gut organisierte und machtvolle Lobbygruppen ist (Becker et al. 2015: 12f.). Die EU zeichnet sich dabei durch eine "strategische Selektivität“ (Jessop 2002: 40) zugunsten der Kapitalseite, speziell transnationaler Unternehmen, aus (Becker et al. 2015: 13). Weil TTIP (und CETA) als Blaupausen für weitere zukünftige Handelsabkommen gelten, bilden diese die Grundlage für den Versuch, Gesetze auf nationalstaatlicher Ebene auszuhöhlen und unter asymmetrischen Kräfteverhältnissen neu zu gestalten.

Um TTIP also adäquat diskutieren und analysieren zu können, ist es notwendig darauf hinzuweisen, dass es bei dem geplanten Abkommen um sehr viel mehr geht als um ökonomische Auswirkungen von Handelsbeziehungen. Neben der geplanten Reduktion tarifärer und nicht-tarifärer Handelsbarrieren, von denen die Befürworter*innen entsprechende ökonomische Effekte in Aussicht stellen (EK 2015a), dreht sich der entscheidende Teil der Verhandlungen um Themen, bei denen demokratisch legitimierte Entscheidungsgremien Unternehmensinteressen untergeordnet werden sollen. Konkret werden öffentliche Dienstleistungen zur Disposition gestellt (Fritz 2015), zukünftige Liberalisierungsmaßnahmen vereinfacht (Soukup 2014), die Position von Unternehmen gegenüber demokratisch legitimierten Interessensvertretungen gestärkt und unter anderem ein einseitiges Klagerecht ausschließlich für multinationale Unternehmen entworfen (Eberhardt 2014a). Mit dem Fokus auf diese heiklen Bereiche von TTIP bietet sich ein weiterer Ansatzpunkt, um die 
Abbildung 1: ISDS-Klagen österreichischer Unternehmen

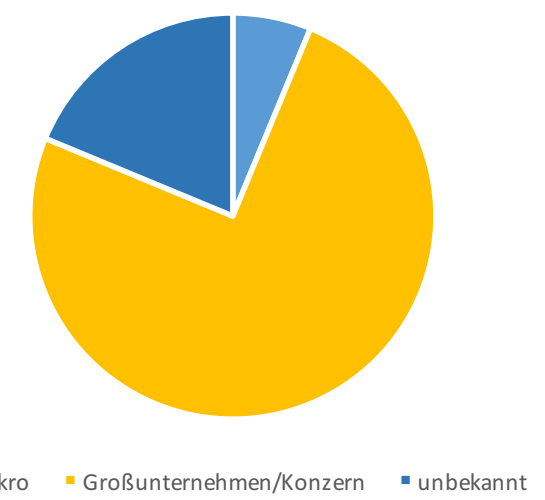

Quelle: Eigene Darstellung - siehe Appendix A1

Interessen hinter TTIP sichtbar zu machen. Dazu soll im Folgenden ein genauerer Blick auf bekannte Fälle geworfen werden, bei denen österreichische Unternehmen Staaten klagen. Nämlich über die sogenannten Investment to State Dispute Settlement (ISDS) Klagen.

Betrachtet man die bisher bekannten Klagen ${ }^{11}$ österreichischer Unternehmen auf vermeintliche Gewinnverluste (siehe Appendix A1), lässt sich auch hier klar aufzeigen, dass beinahe ausschließlich Großkonzerne bestehende Investitionsschutzabkommen genutzt haben. Die vorhandenen Erfahrungen mit österreichischen ISDS-Klagen zeigen, dass zu 94 Prozent Großkonzerne von dem einseitigen Klagerecht Gebrauch gemacht haben.

Es zeigt sich somit, dass auch bei jenen Bereichen von TTIP, die am eindeutigsten Kapitalinteressen stärken und dabei demokratische Strukturen untergraben, transnational agierende Konzerne am meisten profitieren.

\section{Diskussion}

Betrachtet man den politischen Kampf rund um TTIP als Kampf um Hegemonie, so lassen sich zwei Konfliktachsen aufzeigen: erstens der Widerstand der Zivilgesellschaft ${ }^{12}$ gegen das geplante Abkommen und zweitens ein Konflikt zwischen divergierenden Kapitalinteressen. Diese Kapitalinteressen bestehen zwischen europäisch orientierten Unternehmen, die überwie-

11 Eine Darstellung findet sich unter http://derstandard.at/2000045567416/Schiedsgerichte-Oesterreichs-Firmen-legen-sich-mit-Staaten-an [September 2016].

12 z.B. das europaweite Bündnis „Stop-TTIP“ www. stop-ttip.org, der österreichische Gewerkschaftsbund (ÖGB) und viele andere. gend als KMU klassifiziert werden können, und zwischen großen, global orientierten Unternehmen, die als wesentliche Lobbyisten für TTIP in Erscheinung treten und den internationalen Handel dominieren.

Versteht man TTIP als Fortführung neoliberaler Politik in der EU, dann spiegelt sich ein Trend wider, der bereits seit den 1980ern zu beobachten ist, nämlich, dass transnationale Konzerne maßgeblich den europäischen Integrationsprozess beeinflussen konnten und sich für die Umsetzung neoliberaler (Handels-) Politik einsetzten (Apeldoorn 2006; Bieling 2010). Dabei wurde durch die Globalisierung der Handelsbeziehungen und die dabei implementierte Ausweitung des Wettbewerbes auf globale Märkte ein Diskurs über einen vermeintlichen Sachzwang konstruiert, welcher zur Durchsetzung verschiedenster politischer Maßnahmen herangezogen wurde und wird (Jäger 2006, Katzmayr 2006, Huffschmidt 2006).

Neben den für Arbeiter ${ }^{\star}$ innen unvorteilhaften Konsequenzen der neoliberalen Politik (z.B. Boyer 2013) sehen sich nun auch lokal orientierte Kapitalfraktionen, nämlich vorwiegend KMU, durch die Orientierung hin zu mehr Wettbewerbsdruck und durch die geplante Reform staatlicher Vergabepraxen bedroht. Allerdings beschränkt sich der Widerstand der KMU im Moment noch auf einen Widerstand gegen TTIP. Für diesen können KMU somit eine wichtige Rolle spielen, sofern sie ihre eigenen Interessen weiterhin durchzusetzen versuchen. In Hinblick auf größere politische Projekte, z.B. eine Koalition gegen die herrschende neoliberale Hegemonie, sind die Rolle und das Potential von KMU als politischen Akteuren jedoch sehr unklar. 


\section{Literatur}

Apeldoorn, B. van (2006): The Transnational Political Economy of European Integration. In: Underhill, G. / Stubbs, R. (Hg.): Political Economy and the Changing Global Order. Canada: Oxford University Press, 306-316.

Apeldoorn, B. van (2014): The European capitalist class and the crisis of its hegemonic project. Socialist Register 2014: Registering Class,50, 189-206.

Becker, J. / Bösch, V. / Brait, R. / Feigl, G. / Orischnig, T. / Poyntner, P. / Schultheiss, J. (2015): Einleitung: Politische Ökonomie Österreichs - Kontinuitäten und Wandel seit dem EU-Beitritt. In: BEIGEWUM (Hg.)(2015): Politische Ökonomie Österreichs. Kontinuitäten und Veränderungen seit dem EU-Beitritt. Wien: Mandelbaum, 7-34.

Beck, S. / Scherrer, C. (2014): Das transatlantische Handelsund Investitionsabkommen (TTIP) zwischen der EU und den USA. Arbeitspapier 303, Hans Böckler Stiftung.

Bhaskar, R. (2008): A Realist Theory of Science. Sweden: ScandBook AB.

Bieling, H. (2007): Die Konstitutionalisierung der Weltwirtschaft als Prozess hegemonialer Verstaatlichung - Staatstheoretische Reflexionen aus der Perspektive einer neogramscianischen Internationalen Politischen Ökonomie. In: Buckel, S. / Fischer-Lescano, A. (Hg.)(2007): Hegemonie gepanzert mit Zwang. Baden-Baden: Nomos Verlagsgesellschaft, 143-158.

Bieling, H. (2010): Die Globalisierungs- und Weltordnungspolitik der Europäischen Union. VS Verlag.

Bieling, H. / Jäger, J. / Ryner, M. (2015): Regulation Theory and the Political Economy of the European Union. JCMS: Journal of Common Market Studies, 2015, 1-17.

Boyer, R. (2013): The Present Crisis. A Trump for a Renewed Political Economy. Review of Political Economy, 25 (1), 1-38.

Cernat, L. / Norman-López, A. / T-Figueras, A. (2014): SMEs are more important than you think! Challenges and opportunities for EU exporting SMEs. Online: http:// trade.ec.europa.eu/doclib/docs/2014/september/tradoc_152792.pdf [01.08.2015]

Corporate Europe Observatory (2013): European Commission preparing for EU-US trade talks: 119 meetings with industry lobbyists. Online: http://corporateeurope.org/ trade/2013/og/european-commission-preparing-eu-ustrade-talks-119-meetings-industry-lobbyists [01.08.2015]

Corporate Europe Observatory (2015): TTIP talks: big business buys its way into policy processes. Online: http:// corporateeurope.org/power-lobbies/2015/02/ttip-talksbig-business-buys-its-way-policy-processes [01.10.2015]

Demirović, A. (2007): Politische Gesellschaft - zivile Gesellschaft. Zur Theorie des Integralen Staates bei Antonio Gramsci. In: Buckel, S. / Fischer-Lescano, A. (Hg.) (2007): Hegemonie gepanzert mit Zwang. Baden-Baden: Nomos Verlagsgesellschaft, 21-40.
Eberhardt, P. (2014a): Investitionsschutz am Scheideweg. TTIP und die Zukunft des globalen Investitionsrechts. Friedrich Ebert Stiftung.

Eberhardt, P. (2014b): Kampf gegen die transnationale Verfassung der Konzerne: Fünf Thesen zur Debatte um die Investorenrechte im EU-USA-Freihandelsabkommen. Kurswechsel 2/2014,81-85.

Elsner, R. (2015a): TTIP für Kleinunternehmen? Eine Lüge! Online: http://blog.campact.de/2015/05/ttip-fuer-kleinunternehmen-eine-luege/ [01.10.2015]

Elsner, R. (2015b): Five questions SME businesses need to ask themselves about TTIP. Online: http://faireconomyalliance.eu/wp-content/uploads/2017/o2/Fivequestions20151205.pdf [03.08.2015]

Europäische Kommission (2015a): What is TTIP about? Online: http://ec.europa.eu/trade/policy/in-focus/ttip/ about-ttip/ [05.08.2015]

Europäische Kommission (2015b): Small and Medium Sized Enterprises and the Transatlantic Trade and Investment Partnership. Online: http://trade.ec.europa.eu/doclib/ docs/2015/april/tradoc_153348.pdf [05.08.2015]

Europäische Kommission (2015c): Better Regulation. Online: http://ec.europa.eu/smart-regulation/index_en.htm [08.08.2015]

Europäische Kommission (2015d): Annual Report on European SMEs 2012-2013. Online: http://ec.europa.eu/DocsRoom/documents/16106/attachments/1/translations [05.02.2015]

Europäische Kommission (2015e): The top 10 myths about TTIP. Online: http://trade.ec.europa.eu/doclib/ docs/2015/march/tradoc_153266.pdf [05.10.2015]

Francois, J. / Manchin, M. / Norberg, H. / Pindyuk, O. / Tomberger, P. (2013): Reducing Trans-Atlantic Barriers to Trade and Investment. CEPR, London. Online: http://trade.ec.europa.eu/doclib/docs/2013/march/tradoc_150737.pdf [04.08.2015]

Fritz, T. (2015): Public Services under Attack. TTIP, CETA and the secretive collusion between business Lobbyists and trade negotiators. Published by Association Internationale de Techniciens, Experts et Chercheurs (AITEC), Corporate Europe Observatory (CEO), European Federation of Public Services Unions (EPSU), Instytut Globalnej Odpowiedzialności (IGO), Transnational Institute (TNI), Vienna Chamber of Labour (AK Vienna), and War on Want.

Gill, S. (1995): Globalisation, Market Civilisation, and Disciplinary Neoliberalism. Millenium, Journal of International Studies, 24(3), 339-423.

Gramsci, A. (1991): Gefängnishefte. Kritische Gesamtausgabe. Hg. Klaus Bochmann und Wolfgang Fritz. Hamburg: Argument-Verlag.

Grumiller, J. / Theurl, S. / Strickner, A. / Basowski, R. / Krämer, M. (2015): Was bedeutet TTIP für kleinere und mittlere Unternehmen? Online: http://www.attac.at/ kampagnen/ttip-ceta-co-stoppen/kmu-gegen-ttip.html [05.10.2015] 
Huffschmid, J. (2006): Ökonomische Analyse der Standortkonkurrenz. In: ATTAC (Hg.)(2006): Zwischen Konkurrenz und Kooperation. Analysen und Alternativen zum Standortwettbewerb. Wien: Mandelbaum Verlag.

Jäger, J. (2006): Globaler Standortwettbewerb: Realität oder Diskurs? In: ATTAC (Hg.)(2006): Zwischen Konkurrenz und Kooperation. Analysen und Alternativen zum Standortwettbewerb. Wien: Mandelbaum Verlag.

Jessop, B. (2002): The Future of the Capitalist State. Cambridge.

Katzmayr, M. (2006): Der Standortwettbewerb in wirtschaftsgeschichtlicher Betrachtung. In: ATTAC (Hg.) (2006): Zwischen Konkurrenz und Kooperation. Analysen und Alternativen zum Standortwettbewerb. Wien: Mandelbaum Verlag, 7- 21.

Krugman, P. (2009): International Economics. Theory \& Policy. Pearson, Addison Wesley.

Laclau, E. /Mouffe, C. (2006): Hegemonie und radikale Demokratie. Zur Dekonstruktion des Marxismus. 3. Aufl., Wien.

Mayer, T. / Ottaviano, G. (2008): The Happy Few: The Internationalisation of European Firms. Intereconomics, May/ June 2008, 135-148.

OECD TEC (2015): Trade by enterprise characteristics data. Datenbank. Online: http://www.oecd.org/std/its/tradeby-enterprise-characteristics.htm [10.08.2015]

Raza, W. / Grumiller, J. / Taylor, L. / Tröster, B. / Arnim, R. (2014): ASSESS_TTIP. Assessing the Claimed Benefits of the Transatlantic Trade and Investment Partnership. Online: http://guengl.eu/uploads/plenary-focus-pdf/ASSESS_TTIP.pdf [12.08.2015]

Schmiemann, M. (2009): SMEs were the main drivers of economic growth between 2004 and 2006. Online: http:// ec.europa.eu/eurostat/web/products-statistics-in-focus/-/KS-SF-09-071 [01.08.2015]

Schulz, M. (2015): Transatlantische Handels- und Investitionspartnerschaft TTIP - neue Möglichkeiten für KMU? Eine Veranstaltung am 26. Februar 2015 in Brüssel. Online: https://www.wko.at/Content.Node/iv/presse/wkoe_presse/presseaussendungen/pwk_145_15_Schultz:-Freihandelsabkommen-TTIP-bietet-Chancen.html [01.08.2015]

Soukup, N. (2014): Das EU-USA-Handelsabkommen TTIP: Ein neoliberales Projekt und seine Konsequenzen für öffentliche Interessen. WISO, 3/2014.

Theurl, S. / Grumiller, J. (2016): Why are SMEs in Austria taking a stance against TTIP? A qualitative study of the 'SMEs against TTIP' campaign. Online: http://www.oefse.at/fileadmin/content/Downloads/tradeconference/ Theurl_-_Grumiller_-_2016_-_Qualitative_study_-_ SMEs_and_TTIP.pdf [15.11.2016]

UNCTAD (2015): Dataset - ISDS Klagen. Online: http:// unctad.org/en/Pages/DIAE/ISDS.aspx [15.08.2015]

Wallerstein, I. (1979): Class Conflict in the World Economy. In: Wallerstein, I. (1979): The Capitalist World Economy. Cambridge: Cambridge University Press.
Weltbank (2015): Data-set ISDS Klagen. Online: https://icsid.worldbank.org/en/Pages/cases/searchcases.aspx [15.08.2016] 


\section{Appendix A 1}



momentum QUARTERLY 2017 | Vol. 6 (1) Zeitschrift für Sozialen Fortschritt · Journal for Societal Progress 